\title{
Comparison between Inferior Turbinate Reduction Surgery and Continued Medical Treatment in Refractory Allergic Rhinitis Patients with Nasal Obstruction
}

\author{
Subash C. ${ }^{1}$, Lingaraju N. ${ }^{2}$, Vijay S. Jamakhandi ${ }^{3}$ \\ 1,2,3 Department of ENT, Mysore Medical College and Research Institute, Mysore, Karnataka, India.
}

\section{ABSTRACT}

\section{BACKGROUND}

Allergic rhinitis is a widely spread disease that results from an IgE-mediated hypersensitivity reaction of the nasal mucosa to inhaled allergens. It is predominantly treated by allergen avoidance and medical treatment, but when these methods fail to control symptoms then surgery to the inferior turbinate of nose is performed. Objectives of the study were to assess the effectiveness of inferior turbinate surgery on unrelieved or partially relieved nasal obstruction in patients after maximal continued medical management of proven allergic rhinitis, compare the results using different surgical techniques and measure short and long-term results.

\section{METHODS}

This prospective study enrolled patients who had undergone inferior turbinoplasty. All patients were grouped and tested for obstruction of nose, running nose, excessive sneezing, alteration of smell, change in voice due to nasal obstruction, snoring history, and itching of nose. The last follow up of all patients was done at 90 days after surgery. the study was conducted in a tertiary referral center of Mysuru, among 90 patients with refractory allergic rhinitis who had substantial mucosal hypertrophy of the inferior turbinates. 45 patients had undergone inferior turbinate reduction surgeries and 45 patients were on continued medical treatment with follow-up on 7 , 30 , and 90 days after surgery.

\section{RESULTS}

In this study the age of patients ranged from 18 to 50 years. $71 \%$ patients improved significantly. On comparison there was improvement in the condition of patient over time in all groups considering the symptom score. Statistically significant difference in improvement was noted within groups over a period of time, that is before treatment to 7, 30, 90 days after treatment. Whereas on comparing symptoms score between groups, no statistically significant association was found. However, the group which underwent submucous resection has comparatively better results, followed by group with continued medical treatment, group which underwent turbinectomy, and lastly group which underwent out fracturing of inferior turbinates.

\section{CONCLUSIONS}

Our results suggest that inferior turbinate reduction surgeries are effective for decreasing total nasal resistance and improving quality of life in patients with refractory allergic rhinitis who have inferior turbinate hypertrophy with nasal obstruction and all other symptoms.

\section{KEY WORDS}

Allergic Rhinitis, Inferior Turbinate Reduction Surgery, Turbinoplasty, Sub-Mucosal Resection.
Corresponding Author: Dr. Vijay S. Jamakhandi, Department of ENT, OPD Block, K R Hospital, Irvin Road, Mysore - 570001, Karnataka, India.

E-mail: vijaysj24@gmail.com

DOI: $10.14260 /$ jemds/2020/871

How to Cite This Article:

Subash C, Lingaraju N, Jamakhandi VS. Comparison between inferior turbinate reduction surgery and continued medical treatment in refractory allergic rhinitis patients with nasal obstruction. J Evolution Med Dent Sci 2020;9(52):3988-3991, DOI: 10.14260/jemds/2020/871

Submission 12-09-2020,

Peer Review 05-11-2020,

Acceptance 11-11-2020,

Published 28-12-2020.

Copyright (C) 2020 Subash C. et al. This is an open access article distributed under Creative Commons Attribution License [Attribution 4.0 International (CC BY 4.0)] 


\section{BACKGROUND}

Allergic rhinitis is an inflammatory disease of the upper respiratory tract and one of the most common problems seen in outpatient practice affecting around $20-30 \%$ of the Indian population and out of that $15 \%$ to $17 \%$ population subsequently develop asthma. Allergic rhinitis is defined as an inflammatory, IgE-mediated disease characterized by nasal congestion, rhinorrhoea (watery discharge), sneezing, and / or nasal itching. It can also be defined as inflammation of the inside lining of the nasal mucosa that occurs when a person inhales something (allergen), he or she is allergic to, such as animal dander or pollen. Allergic rhinitis symptoms result in sleep problems, fatigue, mood disturbances, and reduction in cognitive function that impairs quality of life. There may be associated conjunctivitis, postnasal drip, (eustachian tube) ET dysfunction, otitis media, sinusitis \& in children; dental anomalies \& facial deformities also. ${ }^{1}$

Nasal obstruction is a common symptom that is because of hypertrophy of inferior turbinates. Inferior turbinate hypertrophy due to venous sinusoid engorgement is a major contributing factor to obstruction at nasal valve level as well as inferior part of nasal cavity. The main determinants of nasal resistance are the anterior and inferior portions of the lower turbinates which can be dealt surgically. The physiological approach to inferior turbinate reduction surgery is based on Poiseuille's Law which states that "the laminar flow rate of air along a pipe is proportional to the fourth power of its radius and hence a small change in the inferior turbinate size will dramatically affect the nasal airflow resistance". Surgical intervention drastically improves the dynamic competence of the nasal cavity airway by addressing the fixed obstruction and alleviates symptoms by reducing the effects of oedematous mucosa. Allergic rhinitis may be classified based on $^{1}$ the temporal pattern of exposure to a triggering allergen, such as seasonal (e.g., pollens, grains), perennial / year-round (e.g., dust mites), or episodic (environmental from exposures not encountered before e.g., visiting a home with pets); ${ }^{2}$ frequency of symptoms; and ${ }^{3}$ severity of symptoms. ${ }^{4}$

Definitive treatment of allergic rhinitis is avoidance of allergens. Medical management include intranasal topical steroids, H1-antihistamines and leukotriene-receptor antagonists. Treatment, either medical management or surgical management of the inferior turbinate is required in cases of turbinate hypertrophy where the goal of therapy is to improve the nasal passage airway, to preserve normal nasal mucosal function and to reduce complications. Refractory allergic rhinitis patients or sustained nasal obstruction patients can be offered.

\section{METHODS}

This prospective clinical study was conducted in a tertiary care hospital, Mysuru, between July 2019 to February 2020 on 90 patients of refractory allergic rhinitis i.e. allergic rhinitis not resolved even after medical management of more than 3 months of duration. Patients are selected in the age range of 18 - 50 years with the symptoms like, sneezing, watery discharge, itching nose, headache and nasal obstruction with hypertrophied inferior turbinates on anterior rhinoscopy.
Informed consent of the patients and ethical committee clearance was obtained.

All patients were evaluated by detailed history taking, complete ENT examination with cold spatula test, cotton wisp test, Cottles test and diagnostic nasal endoscopy to exclude deviated nasal septum, nasal polyp, nasal and nasopharyngeal mass, chronic rhinosinusitis, rhinolith. Patients with medical co morbidities like diabetes mellitus, hypertension, bleeding disorders and cardiac disease, past history of nasal surgery were excluded from the study. Diagnostic nasal endoscopic findings in all patients were noted to rule out any other endonasal and nasal pathology other than ITH and nasal patency was assessed at level of internal nasal valve area (narrowest part of nasal cavity) in terms of percentage and tabulated. All patients were evaluated by nasal symptoms score as shown in Table 1. and were graded according to symptoms.

\begin{tabular}{|c|c|c|c|}
\hline Score & Symptoms & $\begin{array}{l}\text { Categories before } \\
\text { Treatment }\end{array}$ & $\begin{array}{l}\text { Categories after } \\
\text { Treatment } \\
\text { (7 / } 30 \text { / } 90 \text { Days) }\end{array}$ \\
\hline 0 & Not Present & Absent (0.0 \%) & $\begin{array}{l}\text { Significant } \\
\text { Improvement }\end{array}$ \\
\hline 1 & Present but not troublesome & Mild (28.9 \%) & $\begin{array}{l}\text { Moderate } \\
\text { Improvement }\end{array}$ \\
\hline 2 & $\begin{array}{l}\text { Present and frequently } \\
\text { troublesome but not disturbing } \\
\text { daily normal activity }\end{array}$ & Moderate (52.2\%) & Mild Improvement \\
\hline 3 & $\begin{array}{c}\text { Present and disturbing daily } \\
\text { normal activity }\end{array}$ & Severe $(18.8 \%)$ & No Improvement \\
\hline \multicolumn{4}{|c|}{ Table 1. Symptom Score ${ }^{3}$} \\
\hline
\end{tabular}

All the patients in different group were tested preoperatively for age, degree of nasal obstruction, snoring history, smell alterations, running nose history and nasal itching. Inferior turbinate was infiltrated with local anaesthesia and placing of cottonoid patties soaked with vasoconstrictor solution until the end of the procedure to control intraoperative bleeding.

Patients are divided into 2 equal groups of 45 each. Group B containing of 19 females and 26 males were continued with medical management i.e. topical nasal steroid spray and oral anti-histamines and leukotriene receptor antagonist. Group A containing 15 females and 30 males were taken for turbinate reduction surgeries after informed consent. 1) 15 patients underwent turbinectomy (A1). 2) 30 patients underwent turbinoplasty. (A2). a) 15 underwent submucous resection (A2a). b) 15 patients underwent out fracture of the inferior turbinate (A2b), after grading according to nasal obstruction score and nasal symptom score. Then the percentage of symptoms improvement is used to assess treatment outcomes after 7, 30, 90 days after surgery.

The steps of inferior turbinate reduction surgery are as follows:

1) Incision in inferior turbinate in its central part at the horizontal level, then removal of the entire mucosa above the incised horizontal part, after making a small mucosal flap.

2) Inferior turbinate was incised in its anterior to posterior direction using turbinectomy scissors and usually one of scissors blade was directed to the mucosa medially, detached from the underlying turbinate bone in the upper part of the inferior turbinate, while the other blade was directed to its meatus, then removing most of the inferior turbinate bone and almost all of its lateral mucosa. 
3) Medial part was removed only below the given incision, because the mucosa above left was used to cover the bony part, which is left, thereby removing $50 \%$ to $60 \%$ of the medial mucosa, $90 \%$ to $100 \%$ of the lateral mucosa.

4) Subsequently, the bone spicules were cut off with a chisel to minimise the bulging of the remaining part of inferior turbinate, and then the bipolar cautery was used to cauterise bleeding points if present, mainly in the tail part of inferior turbinate.

Turbinectomy is a surgical technique which consists of partial or complete resection of the inferior turbinate, performed with the scissors with or without nasal endoscope, removing the soft tissue from the lateral aspect of the inferior turbinate. In case of a submucous resection technique, an incision is performed at the head of the turbinate. Some of the turbinate tissue is excised with the help of a microdebrider and blunt dissector, allowing the mucosa to recover on a smaller turbinate bone. Out fracturing is another surgical technique, perhaps the simplest way to perform surgery for nasal obstruction. It consists of fracturing the bone of the inferior turbinate and pushing it laterally, subsequently maintaining this position with the aid of a nasal packing.

After the surgery all patients in different groups were transferred to the post-operative room and discharged next day without anterior nasal packing. All the patients were told to avoid blowing of nose on the first postoperative day and were told that bleeding from nose in small amount is common. Subsequently on the third day after surgery, patients were told to start nasal washing with normal saline along with sodium bi carbonate. This is to remove the crusts and debris and maintaining normal physiological ph.

\section{Statistical Analysis}

Descriptive statistical analysis has been carried out in the present study. Multivariate test like repeated measure ANOVA was used to compare the symptom scores [subjective \& objective]. Statistical significance was accepted for $\mathrm{p}$ value < 0.05 .

\section{RESULTS}

Table 1 is the symptom diary provided to each patient at 7, 30 and 90-days postoperative period. Turbinectomy includes total and partial turbinectomy. In this study age of patients ranged from 18 to 50 years. Maximum number of cases were between 36 - 45 years ( $30 \%$ ) followed by patients with age > 46 years $(24.4 \%)$ with mean age of 35.64 years. Male predominance was observed accounting for 53 (58.9\%) patients. Based on the ARIA guidelines there were $52.2 \%$ patients with moderate symptoms and remaining were $18.8 \%$ with severe symptoms. The subjective and objective parameters studied were tabulated and interpreted as shown in Table 4. $4.71 \%$ patients improved significantly. On comparison there was improvement in the condition of patient over the time in all groups considering the symptom score. There was statistical significance found in the improvement within groups over the time that is before treatment to 7,30 and 90 days after treatment. Whereas on comparing symptoms score between groups there was no statistical significance association found. However, group which underwent submucous resection has comparatively better results. This was followed by group with continued medical treatment, group which underwent turbinectomy, group which underwent out fracturing of inferior turbinates. 2 patients developed synechia after 1 month and 1 patient developed intra-operative bleed.

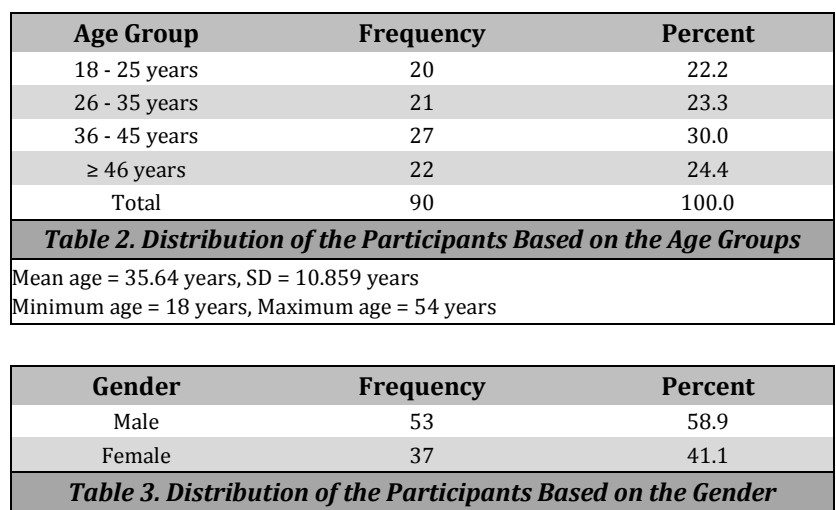

\begin{tabular}{|cccccc|}
\hline GroupParameters & $\begin{array}{c}\text { Before } \\
\text { Treatment }\end{array}$ & $\begin{array}{c}\text { 7 Days after } \\
\text { Treatment }\end{array}$ & $\begin{array}{c}\text { Days after } \\
\text { Treatment }\end{array}$ & $\begin{array}{c}\text { Days after } \\
\text { Treatment }\end{array}$ \\
\hline Group & Mean & 1.96 & 1.29 & 0.62 & 0.33 \\
A & SD & 0.706 & 0.626 & 0.650 & 0.522 \\
Group & Mean & 1.84 & 1.13 & 0.60 & 0.29 \\
B & SD & 0.673 & 0.815 & 0.688 & 0.506 \\
Group & Mean & 2.07 & 1.47 & 0.67 & 0.33 \\
A1 & SD & 0.704 & 0.640 & 0.617 & 0.617 \\
Group & Mean & 1.90 & 1.20 & 0.60 & 0.33 \\
A2 & SD & 0.712 & 0.610 & 0.675 & 0.479 \\
Group & Mean & 1.93 & 1.20 & 0.53 & 0.27 \\
A2a & SD & 0.704 & 0.676 & 0.640 & 0.458 \\
Group & Mean & 1.87 & 1.20 & 0.67 & 0.40 \\
A2b & SD & 0.743 & 0.561 & 0.724 & 0.507 \\
\hline \multicolumn{5}{c}{ Table 4. Mean and SD of Parameters Studied } \\
\hline
\end{tabular}

\section{DISCUSSION}

Successful surgical treatment of hypertrophied inferior turbinates requires effective reduction of tissue (its volume) with preservation of turbinate mucous and ciliary function. If too little tissue is removed, then the obstruction will not get cured. If mucociliary function is impaired by removing the excess mucosa, patients will report crusting and nasal obstruction. 4 Patients with inferior turbinate hypertrophy with allergic rhinitis with nasal obstruction who did not get relieved with medical therapy will benefit from surgical treatment. Even today, clinical experience shows that the success of the nasal functional operation depends on fallowing; Allergic rhinitis + (group I) Allergic rhinitis - (group II) No improvement, moderate improvement, total improvement, degree of snoring improvement. Allergic rhinitis + (group I) no improvement moderate improvement total improvement, degree of improvement in sneezing, pruritus, and rhinorrhoea. In many cases, on how the surgeon approaches the nasal turbinate hypertrophy. Here are several techniques to reduce the turbinates, and according to Mabry et al., the surgeon must know all the available techniques and use them in each case, as required. 5

Most of the patients will not respond to medical management alone. Nasal obstruction is the main symptom in allergic rhinitis with inferior turbinate hypertrophy for main 
visit to ENT OPD in present days. In refractory cases patients will be offered turbinate reduction surgery after failure of continued medical management. Chronic nasal obstruction is main cause for chronic respiratory infection, sinus infection, decreased smell perception and sleep alteration causing the significant morbidity. Allergic inflammation causes venous engorgement of the especially inferior turbinate tissue and mucosa \& the associated inflammation of the mucosal lining as well.

In allergic rhinitis, the mucosal epithelium of the inferior turbinate has been regarded as the main site for IgE mediated inflammation \& nasal accumulation of eosinophils. In most of the time contact area for allergen is anterior most part of the inferior turbinate \& deposition in these areas results in localized inflammation stemming from submucosal structures and blood vessels. ITH (inferior turbinate hypertrophy) usually is due to swelling of submucosa caused by dilatation of venous sinusoids in submucous levels. Significant cases respond to antihistamines or local decongestant, however; occasionally ITH is due to submucous fibrosis rendering the turbinate incapable of decongestion and hence need turbinate reduction surgery.

At 3 months postoperatively, in the submucous resection group, there was improvement in nasal obstruction, discharge, nasal itching, excessive sneezing, chronic headache as shown in table 4 similar to results observed by Datta R. K., Ramya B, Vinay SR et al. ${ }^{3}$ This study showed similar findings. The advantage of inferior turbinate reduction surgery are not only limited to nasal obstruction but also it relieves from symptoms like nasal discharge and excessive sneezing. The main aim of inferior turbinate reduction surgery is to improve the nasal cavity air circulation. However, as turbinoplasty involved in reduction of inferior turbinate mass leads to decrease in exposed part of mucosa to outside environment for nasal allergen and other allergic stimuli and it also reduces gland tissue in inferior partial turbinate that is the reason for improving the symptoms like watery discharge and other rhinitis symptoms. ${ }^{5}$

A variety of surgical procedures are performed for managing ITH, but there is no completely effective therapy. The main aim of turbinate surgery has to be preservation of well-functioning mucosa, along with creation of sufficiently large air space in nasal cavity to ensure the humidification and purification of air and maintenance of a physiological airway resistance. Any method should be judged by two basic criteria: Efficacy of the technique in reducing nasal obstruction and its ability to preserve the nasal mucosa. It is found that more occupational exposure to pollutants in Indian setting, increases the chances of allergic rhinitis induced inferior turbinate hypertrophy in males. ${ }^{6}$ The main aim of surgery of inferior turbinate reduction surgery is to minimize allergen effect by reducing bulky inflammatory tissue or inducing scar formation, while enhancing patency of nasal fossa or cavity by reducing the size of the inferior turbinate but preserving the physiological mucosal function of the turbinate such as humidification \& temperature regulation of inspired air.

\section{CONCLUSIONS}

Surgical techniques like submucosal resection and partial inferior turbinectomy are not only effective in reducing the nasal obstruction in allergic rhinitis due to hypertrophied inferior turbinates but also reduce other symptoms like rhinorrhoea, sneezing, itching and headache. Our results suggest that inferior turbinate reduction surgeries are effective for decreasing nasal obstruction and resistance, improving quality of life in patients with refractory allergic rhinitis who have inferior turbinate hypertrophy with nasal obstruction.

Data sharing statement provided by the authors is available with the full text of this article at jemds.com.

Financial or other competing interests: None.

Disclosure forms provided by the authors are available with the full text of this article at jemds.com.

\section{REFERENCES}

[1] Varshney J, Varshney H. Allergic rhinitis: an overview. Indian J Otolaryngol Head Neck Surg 2015;67(2):143-9.

[2] Seidman MD, Gurgel RK, Lin SY, et al. Clinical practice guideline: allergic rhinitis. Otolaryngol Head Neck Surg 2015;152(1S) S1-43.

[3] Datta RK, Ramya B, Vinay SR. Comparative study between partial inferior turbinectomy and submucosal diathermy for treatment of inferior turbinate hypertrophy due to allergic rhinitis. International Journal of Otorhinolaryngology and Head and Neck Surgery 2018;4(2):362-7.

[4] Dhulipalla S. Comparative study of response through reduction in the size of hypertrophied inferior turbinate causing nasal obstruction by different surgical modalities: a prospective study. Indian J Otolaryngol Head Neck Surg 2015;67(1):56-9.

[5] Hamerschmidt R, Hamerschmidt R, Moreira ATR, et al. Comparison of turbinoplasty surgery efficacy in patients with and without allergic rhinitis. Braz J Otorhinolaryngol 2016;82(2):131-9.

[6] Chavan SS, Khond AD, Saple P, et al. Comparative study between diathermy and microdebrider for inferior turbinate reduction surgeries. Rhinology Online 2020;3:1-7. 